University of Nebraska - Lincoln

DigitalCommons@University of Nebraska - Lincoln

Faculty Publications, Department of Physics and Astronomy

Research Papers in Physics and Astronomy

$3-2002$

\title{
Electrons, Stern-Gerlach Magnets, and Quantum Mechanical Propagation
}

Herman Batelaan

University of Nebraska - Lincoln, hbatelaan@unl.edu

Follow this and additional works at: https://digitalcommons.unl.edu/physicsfacpub

Part of the Quantum Physics Commons

Batelaan, Herman, "Electrons, Stern-Gerlach Magnets, and Quantum Mechanical Propagation" (2002). Faculty Publications, Department of Physics and Astronomy. 121.

https://digitalcommons.unl.edu/physicsfacpub/121

This Article is brought to you for free and open access by the Research Papers in Physics and Astronomy at DigitalCommons@University of Nebraska - Lincoln. It has been accepted for inclusion in Faculty Publications, Department of Physics and Astronomy by an authorized administrator of DigitalCommons@University of Nebraska Lincoln. 


\title{
Electrons, Stern-Gerlach magnets, and quantum mechanical propagation
}

\author{
H. Batelaan ${ }^{\text {a) }}$ \\ Behlen Laboratory of Physics, University of Nebraska-Lincoln, Lincoln, Nebraska 68588-0111
}

(Received 15 August 2001; accepted 20 December 2001)

\begin{abstract}
Quantum corrections to Newton's equations are obtained and used to illustrate that classical dynamics is embedded explicitly in quantum dynamics. Originally, the resultant set of dynamical equations has been used to shed light on quantum chaos. We show that the method can provide insight into the dynamics of free particles and the harmonic oscillator. We then use it to determine whether Stern-Gerlach magnets can be constructed for free electrons. (C) 2002 American Association of Physics Teachers.
\end{abstract}

[DOI: 10.1119/1.1450559]

\section{INTRODUCTION}

One of the cornerstones of quantum mechanics is the Stern-Gerlach effect. An unpolarized beam of silver atoms is passed through a strong magnetic field gradient and splits into two polarized beams. This effect is one of the main reasons to postulate that electrons have spin, in particular spin-1/2.

The dynamical picture of the Stern-Gerlach effect involves the interaction between the magnetic moment $\mu$ associated with spin and the magnetic field gradient. A particle with its projection of spin in the positive/negative $z$ direction will experience a deflection due to the force $F= \pm \mu d B_{z} / d z$ [see Fig. 1(a)].

From this description we might expect that a beam of free electrons would also be split after passing through a SternGerlach magnet. However, Mott, Bohr, and Pauli have shown that this is impossible due to the blurring effect of Lorentz forces on a finite-width beam. ${ }^{2-4}$ A very narrow beam would not suffer from spatially varying Lorentz forces, but would be so badly diffraction limited that it would no longer be a beam. Alternative approaches designed to overcome the Lorentz forces ${ }^{5}$ have been rejected by Pauli. ${ }^{3,6}$ One of these, the longitudinal Stern-Gerlach experiment, originally suggested by Brillouin, ${ }^{5}$ has recently been reexamined. ${ }^{7}$ In this configuration, the magnetic field gradient is aligned with the electron beam, and spin "forward" and spin "backward" electrons passing through the magnet are separated along the direction of propagation [Fig. 1(b)].

To analyze this experimental situation, we cannot resort to the usual semiclassical dynamical picture where the electron motion is treated classically and the spin quantum mechanically. The reason is that to overcome the blurring Lorentz forces, we need to approach the diffraction limit without losing the beam, and have to treat the electron motion quantum mechanically also. We recently analyzed this situation using the Schrödinger equation and obtained the promising result that the blurring is much less than our semiclassical analysis indicated. ${ }^{8}$ As a check on our results, we used a simpler method and found good agreement with the approach using the Schrödinger equation. This method, which is the subject of this paper, seems to have been originally developed for maser theory ${ }^{9}$ and has been used to study quantum chaos. ${ }^{10,11}$ To illustrate the ease of the method, we first address several standard problems that we hope will be useful for educational purposes. We then present the analysis of the longitudinal Stern-Gerlach problem.

\section{THEORY}

Following Sundaram et al., ${ }^{11}$ we write the position operator, $x$, and the momentum operator, $p$, as the sum of their expectation value and quantum correction,

$$
x=\langle x\rangle+\delta x, \quad p=\langle p\rangle+\delta p,
$$

with the commutation relation $[\delta x, \delta p]=i \hbar$. It is useful to express the commutation relation of two arbitrary functions of $x$ and $p$ by making a Taylor expansion about $\langle x\rangle$ and $\langle p\rangle{ }^{12}$ Straightforward algebra yields for two arbitrary functions $F$ and $G$ of $x$ and $p$,

$$
\begin{aligned}
{[F(x, p), G(x, p)]=} & i \hbar\{F(x, p), G(x, p)\}+\frac{1}{2}(\delta x \delta p \\
& +\delta p \delta x)\left(\frac{\partial^{2} F}{\partial x^{2}} \frac{\partial^{2} G}{\partial p^{2}}-\frac{\partial^{2} G}{\partial x^{2}} \frac{\partial^{2} F}{\partial p^{2}}\right) \\
& +\cdots\left\langle\delta x^{2}\right\rangle\left(\frac{\partial^{2} F}{\partial x^{2}}\right. \\
& \left.-\frac{\partial^{2} G}{\partial x \partial p} \frac{\partial^{2} G}{\partial x^{2}} \frac{\partial^{2} F}{\partial x \partial p}\right)-\cdots\left\langle\delta p^{2}\right\rangle \\
& \times\left(\frac{\partial^{2} F}{\partial p^{2}} \frac{\partial^{2} G}{\partial x \partial p}-\frac{\partial^{2} G}{\partial p^{2}} \frac{\partial^{2} F}{\partial x \partial p}\right)+\cdots,
\end{aligned}
$$

where the curly braces \{\} indicate the Poisson bracket. The time evolution of the expectation value of an operator that can be written as a function of $x$ and $p$ can be obtained from the Heisenberg equation. For example, if we let $F(x, p)=p$ and $G(x, p)=H$, we obtain

$$
\frac{d\langle p\rangle}{d t}=\frac{1}{i \hbar}[p, H]=\{p, H\}+\cdots .
$$

Together with $H=p^{2} / 2 m+V(x)$, Eq. (3) gives:

$$
\frac{d\langle p\rangle}{d t}=-\left.\frac{\partial V}{\partial x}\right|_{x=\langle x\rangle}-\left.\frac{1}{2} \frac{\partial^{3} V}{\partial x^{3}}\right|_{x=\langle x\rangle}\left\langle\delta x^{2}\right\rangle+\cdots .
$$

The evolution of $\langle x\rangle,\left\langle\delta x^{2}\right\rangle,\left\langle\delta x^{3}\right\rangle, \ldots,\langle\delta x \delta p\rangle, \ldots$, can be found in a similar manner. The resulting equations to secondorder are given below. No additional calculational difficulties are encountered to obtain expressions for third- and higherorder terms such as $d\left\langle\delta x^{3}\right\rangle / d t$, 
a)

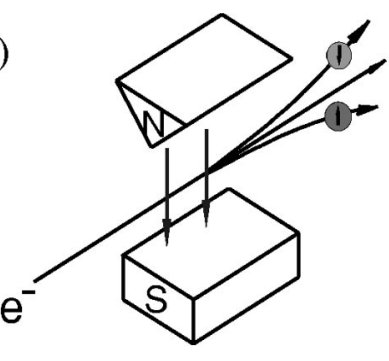

b)

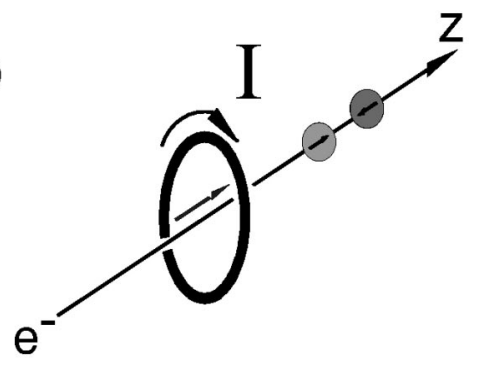

Fig. 1. A schematic view of (a) the traditional Stern-Gerlach magnet and (b) the longitudinal Stern-Gerlach magnet consisting of a current loop. Arrows indicate the magnetic field direction, while the arrows in the "particle" represent spin-up (spin-forward) and spin-down (spin-backward), respectively.

$$
\begin{aligned}
& \frac{d\langle x\rangle}{d t}=\langle p\rangle / m, \\
& \frac{d\left\langle\delta x^{2}\right\rangle}{d t}=(\langle\delta x \delta p\rangle+\langle\delta p \delta x\rangle) / m, \\
& \frac{d\langle\delta x \delta p\rangle}{d t}=\left\langle\delta p^{2}\right\rangle / m-\left.\frac{\partial^{2} V}{\partial x^{2}}\right|_{x=\langle x\rangle}\left\langle\delta x^{2}\right\rangle+\cdots, \\
& \frac{d\langle\delta p \delta x\rangle}{d t}=\left\langle\delta p^{2}\right\rangle / m-\left.\frac{\partial^{2} V}{\partial x^{2}}\right|_{x=\langle x\rangle}\left\langle\delta x^{2}\right\rangle+\cdots, \\
& \frac{d\left\langle\delta p^{2}\right\rangle}{d t}=-\left.(\langle\delta x \delta p\rangle+\langle\delta p \delta x\rangle) \frac{\partial^{2} V}{\partial x^{2}}\right|_{x=\langle x\rangle}+\cdots
\end{aligned}
$$

This set of equations, which can be continued indefinitely, gives the values of $\langle x\rangle,\left\langle\delta x^{2}\right\rangle,\left\langle\delta x^{3}\right\rangle, \ldots$ from which the moments $\langle x\rangle,\left\langle x^{2}\right\rangle, \ldots$ can be obtained.

From the Fourier transform of the probability distribution $|\psi|^{2}$ :

$$
\begin{aligned}
\int e^{i k x}|\psi|^{2} d x & =\sum_{n} \frac{(i k)^{n}}{n !} \int x^{n}|\psi|^{2} d x \\
& \equiv \sum_{n} \frac{(i k)^{n}}{n !}\left\langle x^{n}\right\rangle,
\end{aligned}
$$

we observe that a knowledge of all the moments $\left\langle x^{n}\right\rangle$ allows us to construct the probability distribution $|\psi|^{2}$ through an inverse Fourier transform. The solution of the set of equations (4)-(9) and its higher orders represent the basic method used throughout this paper.

We first indicate some limitations of the method before applying it to several examples. If we are not interested in dynamics but in, say, energy eigenvalues, the method cannot be used. Furthermore, knowing all the moments does not automatically imply that the probability distribution can be calculated. A general method for obtaining the function itself from its moments does not seem to exist. ${ }^{13}$ The method is useful when we are interested in studying the dynamics of a system and the above set of equations either truncates or converges rapidly. That is, the method can be applied when the motion of a system is expected to be close to the classical dynamics. Finally, we note that Eq. (4) can be obtained by making a Taylor expansion of the potential in Ehrenfest's theorem: ${ }^{14}$

$$
\frac{d\langle p\rangle}{d t}=-\left\langle\frac{\partial V}{\partial x}\right\rangle
$$

about the expectation value of the position.

\section{FREE PARTICLE}

What insight can be obtained from Eqs. (4)-(9)? Why not use Schrödinger's equation, which is one differential equation in contrast to the above infinite set of differential equations, most of which have an infinite number of terms? The following question provides a partial answer. For which potentials does the expectation value of the position follow the classical trajectory exactly? One way to obtain the answer is to compare the form of the time evolution of the Wigner equation ${ }^{15}$ to the classical Liouville equation and note that the quantum mechanical corrections introduced in the former involve third- and higher-order spatial derivatives of the potentials. Hence, the desired potential is any potential that has zero third- and higher-order spatial derivatives.

A simpler way of obtaining this result is to note that for any potential of the form $V(x)=a x^{2}+b x+c$, Eqs. (4) and (5) reduce to their classical counterparts, ${ }^{10}$ and quantum corrections involve third- and higher-order spatial derivatives of the potential. Equations (4) and (5) describe the average position and momentum and have the same form as Newton's equation for quadratic potentials. Thus, the expectation value of the position follows its classical counterpart exactly for this form of $V(x)$, and we see that the present approach exemplifies how classical mechanics is embedded in quantum mechanics.

Although free particle propagation and the harmonic potential are discussed in quantum mechanics textbooks (the study of a linear potential is less common, but does exist ${ }^{16}$ ), each potential needs to be studied separately and with differing mathematical approaches. The present approach offers a good opportunity to study, for example, a free particle and the harmonic oscillator from the same viewpoint.

To illustrate this point, consider the spreading of the probability distribution for a free particle. We would like to obtain the time evolution of the width, $d \sqrt{\left\langle\delta x^{2}\right\rangle} / d t$. For a free particle (or particle in a linear and quadratic potential), Eqs. (6)-(9) form a closed set of differential equations with a limited number of terms. From Eq. (9) it is apparent that the width of the momentum distribution will not change with time for a free particle. After substitution of Eqs. (7)-(9) into Eq. (6), we are left with $d^{2}\left\langle\delta x^{2}\right\rangle / d t^{2}=2\left\langle\delta p^{2}\right\rangle_{t=0} / m^{2}$, which is solved by 


$$
\begin{aligned}
\left\langle\delta x^{2}\right\rangle_{t}= & \left\langle\delta x^{2}\right\rangle_{0}+\frac{1}{m}\left[\langle\delta x \delta p\rangle_{0}+\langle\delta p \delta x\rangle_{0}\right] t \\
& +\frac{1}{m^{2}}\left\langle\delta p^{2}\right\rangle_{0} t^{2}
\end{aligned}
$$

where the notation \langle\rangle$_{0}$ means that the term in brackets is evaluated at $t=0$.

We choose a Gaussian wave packet, $|\psi(x, 0)|^{2}$ $=\sqrt{\left(2 / \pi a^{2}\right)} e^{-2 x^{2} / a^{2}}$, as the initial condition with initial widths in position and momentum space, such that the equality for the Heisenberg's uncertainty relation holds,

$$
\Delta x \Delta p=\sqrt{\left\langle\delta x^{2}\right\rangle} \sqrt{\left\langle\delta p^{2}\right\rangle}=\hbar / 2 .
$$

We follow the convention for the definition of width as given in Ref. 17, and obtain $\Delta x=a / 2$. This choice yields the initial conditions,

$$
\left\langle\delta x^{2}\right\rangle_{0}=a^{2} / 4, \quad\langle\delta x \delta p+\delta p \delta x\rangle_{0}=0, \quad\left\langle\delta p^{2}\right\rangle_{0}=\hbar^{2} / a^{2} .
$$

The second initial condition in Eq. (14) can be found by combining the uncertainty principle with the commutation relation $[\delta x, \delta p]=i \hbar$ giving $\langle\delta x \delta p\rangle_{t=0}=i \hbar / 2$ and $\langle\delta p \delta x\rangle_{t=0}=-i \hbar / 2$. With these initial conditions in Eq. (14), the usual spreading of the free wave packet,

$$
\Delta x(t)=\frac{a}{2} \sqrt{1+\frac{4 \hbar^{2} t^{2}}{m^{2} a^{4}}},
$$

is found by substituting Eq. (14) into Eq. (12). ${ }^{17}$ Note that this result is the same for a free particle and a particle in a linear potential.

\section{HARMONIC OSCILLATOR}

To obtain the correspondence between the classical and quantum harmonic oscillator, we can compare their respective probabilities for finding the particle at a position $x$. This probability is peaked around the classical turning points and shows an increasing degree of agreement for higher energy states. ${ }^{18}$ Schrödinger introduced states for which $\langle x\rangle$ and $\langle p\rangle$ follow their oscillating classical counterparts exactly. ${ }^{19} \mathrm{We}$ can use the present method to show that for every initial state, $\langle x\rangle$ and $\langle p\rangle$ will follow their classical counterparts exactly. This result can also be obtained by using the Schrödinger or Heisenberg equation. ${ }^{20}$

To find how the widths evolve in time in the potential $V(x)=\frac{1}{2} m \omega^{2} x^{2}$, we have to choose initial conditions. We start again with a minimum uncertainty state. ${ }^{21}$ If we require that the widths not change in time, then $d\left\langle\delta x^{2}\right\rangle / d t=0$, $d\langle\delta x \delta p\rangle / d t=0$, and $d\langle\delta p \delta x\rangle / d t=0$ for all times. By substitution this requirement leads to $\left\langle\delta p^{2}\right\rangle / m=m \omega^{2}\left\langle\delta x^{2}\right\rangle$. If the rate of change we combine this result with the Heisenberg uncertainty conditions, Eq. (13), we obtain

$$
\begin{aligned}
& \left\langle\delta x^{2}\right\rangle=\hbar / 2 m \omega, \\
& \left\langle\delta p^{2}\right\rangle=\hbar m \omega / 2 .
\end{aligned}
$$

These widths are identical to those of coherent states. ${ }^{22}$ That is, an initial Gaussian wave packet placed in a harmonic potential (not necessarily in the center) will not change its width over time.

If the widths are chosen differently, such that we still have a minimum uncertainty state but not a coherent state, then

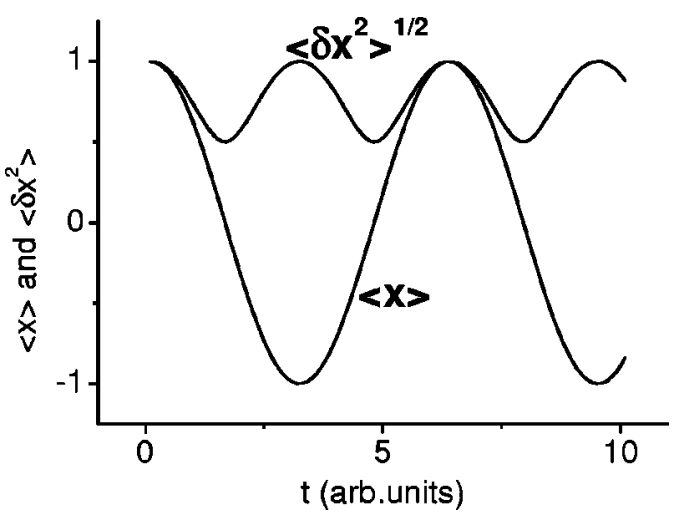

Fig. 2. The quantum average position of a particle, $\langle x\rangle$, in a harmonic oscillator follows its classical counterpart exactly. The width of the probability distribution, $\left\langle\delta x^{2}\right\rangle^{1 / 2}$, oscillates with twice the frequency for a squeezed state.

the widths start to oscillate with the motion. It is easy to show that the solutions of Eqs. (6)-(9) are sinusoidal functions with angular frequency $2 \omega$. Figure 2 shows how the width $\Delta x=\left\langle\delta x^{2}\right\rangle^{1 / 2}$ changes in time. Starting with $\left\langle\delta x^{2}\right\rangle$ $=\hbar / 8 m \omega$ (and accordingly $\left\langle\delta p^{2}\right\rangle=2 \hbar m \omega$ ), the width $\Delta x$ is a minimum whenever the position $\langle x\rangle$ crosses zero, and $\Delta x$ is maximum at the turning points. Minimum uncertainty states with this property are called squeezed states. They are defined by the property that $\Delta x$ (or $\Delta p$ ) is "squeezed" below the width of the coherent state. Simultaneously, $\Delta p$ (or $\Delta x$ ) has to be larger than the width of the coherent state to ensure that the uncertainty relation holds at all times.

How can we now change a coherent state into a squeezed state? One might think that applying an electric field pulse to a charged particle starting in the lowest energy eigenstate of the harmonic oscillator would suffice. The lowest energy eigenstate is a Gaussian wave packet, and kicking the wave packet to one side by an electrical pulse will start the wave packet oscillating in the potential well. However, its width will not change because the strength of the potential, or in other words, $\omega$, did not change. The initial conditions for $\left\langle\delta x^{2}\right\rangle$ and $\left\langle\delta p^{2}\right\rangle$ are still such that $\left\langle\delta x^{2}\right\rangle$ does not change in time; thus, the conditions (16) and (17), which depend on $\omega$ and not on the external pulse, still hold. The way to make a squeezed state is to change the strength of the potential. But how fast do we need to change the potential? One would expect that when the rate of change in the potential, $(1 / V)$ $\times(\partial V / \partial t)$, is small compared to the oscillation frequency of the particle in the potential, the width of the Gaussian wave packet does not change much [see Fig. 3(b)]. By numerically integrating the differential equations governing the evolution of the widths, this result turns out to be the case. When the rate of change in the potential is large compared to the oscillation frequency, the width of the wave packet changes quickly and some squeezing is obtained [see Fig. 3(a)]. The amount of squeezing can be found by numerically integrating Eqs. (6)-(9). Choose for an example, $m=1$, and $V(t)$ $=m \omega^{2} \alpha(t) / 2$, where $\alpha(t)=1+(\arctan (\beta(t-50))+\pi / 2) / \pi$. A slow change in the width can be obtained for $\beta=0.2$, while the fast change can be obtained for $\beta=5$. 

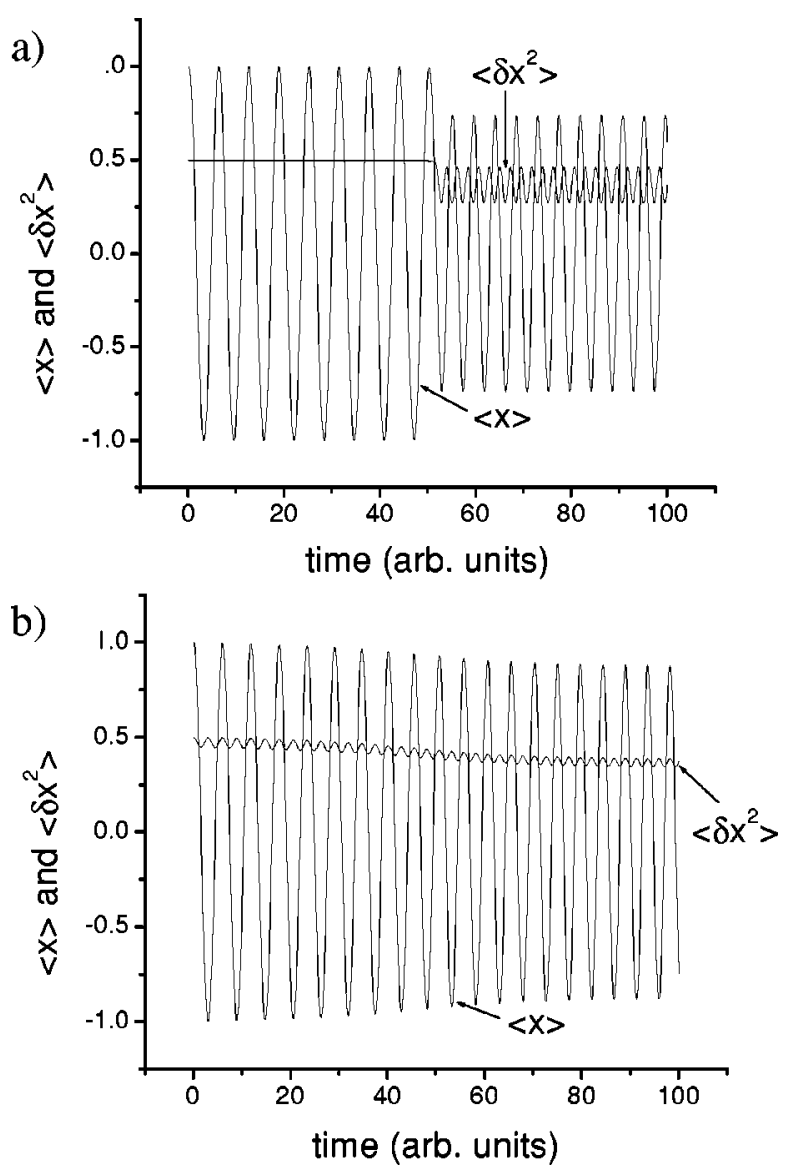

Fig. 3. (a) The abrupt change of the strength of a harmonic potential at $t$ $=50$ changes a coherent state to a squeezed state, as shown by the change in the evolution of the width. Simultaneously, the frequency and the amplitude of the oscillation change. (b) The same amount of change is made, but slowly. Now the change does not change the evolution of the width much.

\section{TUNNELING}

For both the free particle and the harmonic oscillator, the motion of the particle is decoupled from the evolution of its width. In other words, Eqs. (4) and (5) are decoupled from Eqs. (6)-(9). Consider a potential for which this behavior is not the case, which means that at least $\partial^{3} V / \partial x^{3} \neq 0$. The latter condition is satisfied for a one-dimensional tunneling problem with a smooth potential barrier, such as $V(x)$ $=V_{0} \cosh ^{-2}(x / a)$. If we take $a=10^{-9} \mathrm{~m}$ and $V_{0}=3 \mathrm{eV}$, then a particle with the mass of an electron moving with a kinetic energy of about $2.7 \mathrm{eV}$ toward this potential barrier has an appreciable probability of tunneling through this barrier.

Tunneling can be treated exactly for this potential because the wave functions can be obtained analytically. ${ }^{23}$ We can compare this treatment to direct numerical integration of Eqs. (4)-(9) (see Fig. 4). Classically, the momentum of the particle changes sign, while the position returns to its initial value; the particle reflects from the barrier. In the quantum mechanical description, the particle behaves quite differently. The particle first slows somewhat more than in the classical case, and subsequently decelerates less than in the classical case, even before the top of the barrier is reached. We suggest that the deviation from the classical motion can be viewed as the onset of tunneling. To understand this behavior, we may inspect Eq. (4). For a sufficiently small width $\sqrt{\left\langle\delta x^{2}\right\rangle}$, the force is equal to $-\left.(\partial V / \partial x)\right|_{x=\langle x\rangle}$, which is the

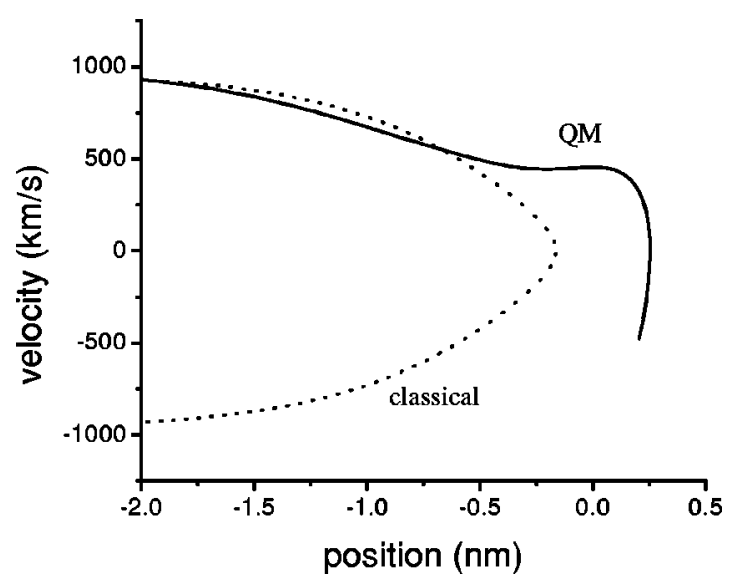

Fig. 4. A particle with the mass of an electron moves into a potential barrier with parameters such that tunneling is not negligible (see the text for details). Its average position does not follow the classical trajectory due to the quantum corrections to the classical force [the second term on the right-hand side of Eq. (4)]. The dotted line indicates the classical trajectory.

classical force, and the particle follows the classical trajectory. For larger widths, the particle samples the force around the average position of $x$. Keeping in mind that Eq. (4) can be obtained by a Taylor expansion of Ehrenfest's theorem, the next term we should look at involves $\partial^{2} V / \partial x^{2}$. This term is $\partial^{2} V /\left.\partial x^{2}\right|_{x=\langle x\rangle} \int x|\psi|^{2} d x$, where the integral is zero due to its antisymmetric argument and thus does not appear in Eq. (4). That is, a change of the force $\left(\propto \partial^{2} V / \partial x^{2}\right)$ does not have an effect because an increase in the force to one side of $\langle x\rangle$ will be canceled by a decrease in the force to the other side of $\langle x\rangle$. The first nonzero correction is proportional to the curvature of the force [the second term on the right-hand side of Eq. (4)].

Another important observation can be made from Fig. 4. The momentum changes abruptly with very little change in the position. This unphysical behavior occurs when it is no longer valid to use the truncated set of differential equations (4)-(9). As soon as tunneling occurs, the wave function splits into a reflected and a transmitted part. To describe this effect, many moments of the probability distribution are needed, and Eqs. (4)-(9) include moments only up to second-order. However, increasing the number of differential equations is not very useful because we do not expect the motion to stay close to the classical trajectory. This example illustrates the limitations of the present method. We now turn our attention to the longitudinal Stern-Gerlach magnet.

\section{THE LONGITUDINAL STERN-GERLACH MAGNET}

An electron in a homogeneous magnetic field can be described in terms of Landau states. ${ }^{24}$ In the Coulomb gauge, it turns out that the effects of the vector potential on a charged particle are the same as those of a harmonic scalar potential. Although interesting in itself, for our purposes it is most important that we can work with two-dimensional harmonic oscillator states instead of Landau states.

Consider an unpolarized electron beam passing through a longitudinal Stern-Gerlach magnet [see Fig. 1(b)]. We choose our quantization axis along the symmetry axis $(z$ axis) of the magnet. We would like to show that the spinforward and spin-backward parts of the beam are separated 
after passing through the magnet along the longitudinal $z$ direction. Such a separation of two spin states along the same axis could only be detected for a pulsed beam. Therefore, imagine our electron beam to consist of two overlapping, incoherent (three-dimensional) Gaussian wave packets propagating toward the magnet along the symmetry axis. The separation between the average positions along the symmetry axis (the $z$ axis) of the packets after they leave the magnet is given by

$$
\Delta z_{\text {spin }}=\iint a_{z} d t^{\prime} d t=\iint \frac{2 \mu_{B}}{m} \frac{\partial B_{z}}{\partial z} d t^{\prime} d t,
$$

where $a_{z}$ is the acceleration along the $z$ axis caused by the magnetic gradient forces, $\pm \mu_{B} \partial B_{z} / \partial z$, on each of the two spins, and $\mu_{B}$ is the Bohr magneton. The first integration gives the velocity and the second integration gives the change in position. Note that for very small changes in velocity, the approximation $a_{z}(z(t))=a_{z}\left(v_{z}(t=0) t\right)$ gives an excellent result. The question that we have to answer is whether the width of the wave packets along the symmetry axis has increased significantly compared to $\Delta z_{\text {spin }}$ after passing through the magnet.

Classically, we have reason to believe that there is some blurring. ${ }^{7}$ An electron entering the magnet off-axis performs a cyclotron motion in the magnetic field. As the electron moves closer to the magnet, the strength of the field increases, and the orbit of the cyclotron motion becomes tighter. This tighter orbit means that the orbital velocity of the electron increases to keep the centrifugal and Lorentz forces balanced. This increase in velocity takes energy from the longitudinal motion of the electron. This effect is called the magnetic bottle effect and slows off-axis electrons in comparison to on-axis electrons.

Quantum mechanically, one may overcome this problem by inserting an electron in the lowest Landau state into the magnet. The orbital angular momentum of this state is zero; thus, no magnetic bottle effect occurs, improving the separation of the two spin states. ${ }^{8}$ In terms of our present method, the question would be formulated this way. Does the transverse width of the wave packet couple to the longitudinal width of the wave packet, as it would for the magnetic bottle effect? The full set of coupled differential equations (see the Appendix) shows that these widths are indeed coupled. But, the range and strength of the magnetic field can be chosen in such a way that the widths follow the change of the magnetic field adiabatically. As the packet enters the magnetic field, it is fairly wide in the transverse direction due to the fact that the magnetic field is weak. After being narrowed in the center of the magnet, it returns to its original width. The spreading of the longitudinal width is the same as that of a free particle and is not influenced by the strongly changing transverse width. Figure 5 shows the results of a numerical integration of the three-dimensional version of Eqs. (21)-(37). The position dependence of the magnetic field is that of a current loop (see the Appendix). We find that the passage of a Gaussian wave packet is nearly adiabatic in agreement with the results of Gallup et al. ${ }^{8}$ An artistic impression of the spin separation is given in Fig. 6.

\section{SUMMARY}

Equations (4)-(9) give some insight into the connection between the quantum mechanical and classical treatment of a
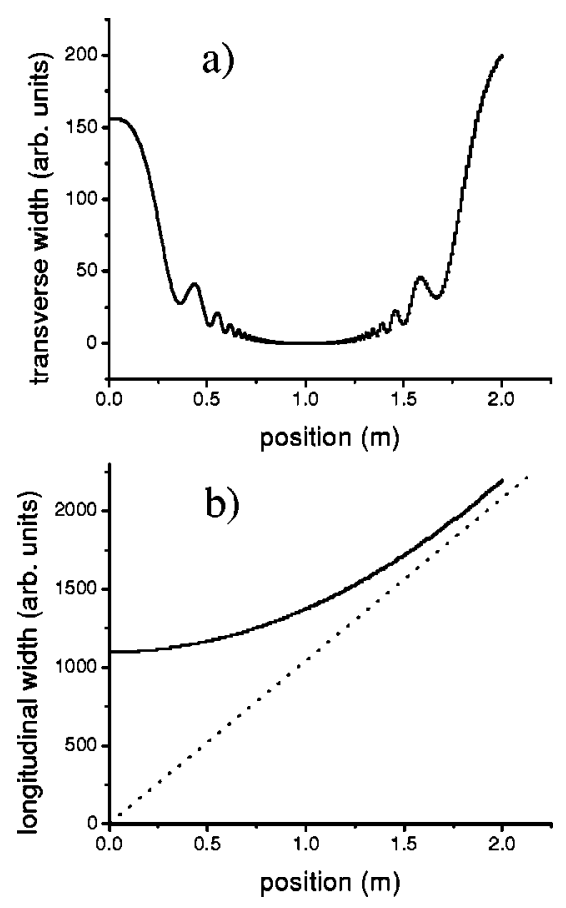

Fig. 5. The width of the probability distribution associated with a particle propagating through a longitudinal Stern-Gerlach magnet. The center of the magnet is situated at $1.0 \mathrm{~m}$. The parameters are the same as in Ref. 8, the electrons follow a 2-m path length that has a midpoint at the center of a $2-\mathrm{cm}$ radius current ring, where the field magnitude is $10 \mathrm{~T}$. The initial electron speed is taken to be $10^{5} \mathrm{~m} / \mathrm{s}$. The splitting obtained is $631 \mu \mathrm{m}$. Although the transverse width in (a) varies drastically, it is close to adiabatic and does not couple to the longitudinal width shown in (b), which evolves in a similar manner as a free particle.

particle in a potential. Some of the usual properties of a free particle and of a particle in a quadratic potential were found. In general, the method is useful when the quantum and classical motion do not deviate strongly. In that case a small set of equations with a limited number of terms is expected to give a reasonable approximation. Not only can the presented

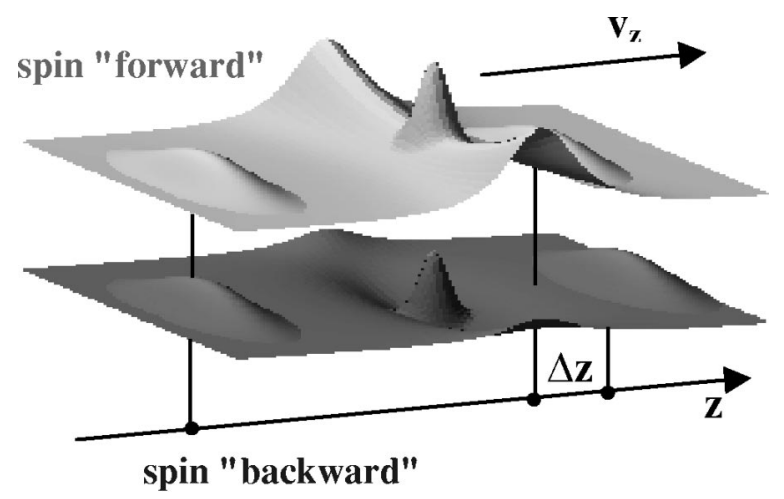

Fig. 6. Two Gaussian probability distributions propagating along the $z$ direction enter a longitudinal Stern-Gerlach magnet, one with spin-forward, the other with spin-backward. In the center of the magnet their transverse width is compressed by the strong magnetic field, while the spin-forward electrons have climbed a potential hill and the spin-backward electrons have descended a potential valley. Emerging from the magnet, the spin-forward electrons have slowed down compared to the spin-backward electrons, and are separated from each other. The transverse width is restored to its original value. The longitudinal width only experienced the spreading of a free particle. For a more rigorous treatment see Gallup et al. (Ref. 8). 
method be applied to consider textbook problems from a different perspective, it can also be used for some current research issues, of which the longitudinal Stern-Gerlach problem is an example.

\section{ACKNOWLEDGMENTS}

The author thanks Emil Sidky, Gordon Gallup, and Dan Freimund for stimulating discussions.

\section{APPENDIX}

The Hamiltonian for an electron passing through the magnetic field of a current loop, that is, the longitudinal Stern Gerlach problem (see Fig. 1), is given in cylindrical coordinates $(\rho, \varphi, z)$ by

$$
H=\frac{p_{\rho}^{2}}{2 m}+\frac{p_{\varphi}^{2}+\frac{1}{4}}{2 m \rho^{2}}+\frac{p_{z}^{2}}{2 m}-\frac{\omega(z)}{2} L_{z}+\frac{1}{8} m \omega^{2}(z) \rho^{2} .
$$

We ignore all spin-dependent terms because the blurring due to magnetic bottle effects are spin independent (see Sec. VI). ${ }^{8}$ The angular momentum in the $z$ direction equals the tangential momentum, $L_{z}=p_{\varphi}$. The Larmor frequency is given by $\omega(z)=q B(z) / 2 m$. The magnetic field, $B(z)$, of the current loop is

$$
B(z)=B_{0}\left(\frac{R}{\sqrt{R^{2}+z^{2}}}\right)^{3},
$$

where $R$ is the loop radius and $B_{0}$ is the magnetic field strength in the center of the loop.

The set of differential equations that describe the average position, momentum, and widths of the probability distribution are given below. Equations (5), (4), (6), (7), and (9) are the one-dimensional versions of the three-dimensional equations (21), (22), (23), (24), and (25), respectively. The coordinates $\rho$ and $\varphi$ lead to additional differential equations required to close the following set of equations so that they can be integrated numerically. Most important for our discussion is that the value $\left\langle\delta z^{2}\right\rangle$ is a measure of the longitudinal width of the wave packet, while $\left\langle\delta \rho^{2}\right\rangle$ is a measure of the transverse width. Additional differential equations include the terms for the tangential momentum, $p_{\varphi}$, and the radial momentum, $p_{\rho}$,

$$
\begin{aligned}
& \frac{d\langle z\rangle}{d t}=\left\langle p_{z}\right\rangle / m, \\
& \frac{d\left\langle p_{z}\right\rangle}{d t}=-\left.\frac{\partial H}{d z}\right|_{x=\langle x\rangle}-\left.\frac{1}{2} \frac{\partial^{3} H}{\partial z^{3}}\right|_{x=\langle x\rangle}\left\langle\delta z^{2}\right\rangle \\
& -\left.\frac{\partial^{3} H}{\partial z^{2} \partial p_{\varphi}}\right|_{x=\langle x\rangle}\left\langle\delta z \delta p_{\varphi}\right\rangle-\left.\frac{1}{2} \frac{\partial^{3} H}{\partial z \partial \rho^{2}}\right|_{x=\langle x\rangle}\left\langle\delta \rho^{2}\right\rangle \\
& -\left.\frac{\partial^{3} H}{\partial z^{2} \partial \rho}\right|_{x=\langle x\rangle}\langle\delta z \delta \rho\rangle, \\
& \frac{d\left\langle\delta z^{2}\right\rangle}{d t}=2\left\langle\delta z \delta p_{z}\right\rangle / m \\
& \frac{d\left\langle\delta z \delta p_{z}\right\rangle}{d t}=\left\langle\delta p_{z}^{2}\right\rangle / m-A\left\langle\delta z^{2}\right\rangle-B\left\langle\delta z \delta p_{\varphi}\right\rangle-C\langle\delta z \delta \rho\rangle,
\end{aligned}
$$

$$
\begin{aligned}
& \frac{d\left\langle\delta p_{z}^{2}\right\rangle}{d t}=-2 A\left\langle\delta z \delta p_{z}\right\rangle-2 B\left\langle\delta p_{z} \delta p_{\varphi}\right\rangle-2 C\left\langle\delta p_{z} \delta \rho\right\rangle, \\
& \frac{d\left\langle\delta z \delta p_{\varphi}\right\rangle}{d t}=\left\langle\delta p_{z} \delta p_{\varphi}\right\rangle / m \\
& \frac{d\langle\delta z \delta \rho\rangle}{d t}=\left\langle\delta z \delta p_{\rho}\right\rangle / m+\left\langle\delta \rho \delta p_{z}\right\rangle / m \\
& \frac{d\left\langle\delta p_{z} \delta p_{\varphi}\right\rangle}{d t}=-A\left\langle\delta z \delta p_{\varphi}\right\rangle-B\left\langle\delta p_{\varphi}^{2}\right\rangle-C\left\langle\delta \rho \delta p_{\varphi}\right\rangle, \\
& \frac{d\left\langle\delta \rho \delta p_{z}\right\rangle}{d t}=\left\langle\delta p_{z} \delta p_{\rho}\right\rangle / m-A\langle\delta z \delta \rho\rangle-B\left\langle\delta \rho \delta p_{\varphi}\right\rangle \\
& -C\left\langle\delta \rho^{2}\right\rangle \\
& \frac{d\left\langle\delta z \delta p_{\rho}\right\rangle}{d t}=\left\langle\delta p_{z} \delta p_{\rho}\right\rangle / m-C\left\langle\delta z^{2}\right\rangle-D\langle\delta z \delta \rho\rangle, \\
& \frac{d\left\langle\delta p_{\varphi}^{2}\right\rangle}{d t}=0 \\
& \frac{d\left\langle\delta \rho \delta p_{\varphi}\right\rangle}{d t}=\left\langle\delta p_{\rho} \delta p_{\varphi}\right\rangle / m \\
& \frac{d\left\langle\delta \rho^{2}\right\rangle}{d t}=2\left\langle\delta \rho \delta p_{\rho}\right\rangle / m \\
& \frac{d\left\langle\delta p_{z} \delta p_{\rho}\right\rangle}{d t}=-A\left\langle\delta z \delta p_{\rho}\right\rangle-B\left\langle\delta p_{\rho} \delta p_{\varphi}\right\rangle-C\left\langle\delta \rho \delta p_{\rho}\right\rangle \\
& -C\left\langle\delta z \delta p_{z}\right\rangle-D\left\langle\delta \rho \delta p_{z}\right\rangle \\
& -E\left\langle\delta p_{z} \delta p_{\varphi}\right\rangle \frac{d\left\langle\delta \rho \delta p_{\rho}\right\rangle}{d t} \\
& =\left\langle\delta p_{\rho}^{2}\right\rangle / m-C\langle\delta z \delta \rho\rangle-D\left\langle\delta \rho^{2}\right\rangle, \\
& \frac{d\left\langle\delta p_{\rho} \delta p_{\varphi}\right\rangle}{d t}=-C\left\langle\delta z \delta p_{\varphi}\right\rangle-D\left\langle\delta \rho \delta p_{\varphi}\right\rangle-E\left\langle\delta p_{\varphi}^{2}\right\rangle, \\
& \frac{d\left\langle\delta p_{\rho}^{2}\right\rangle}{d t}=-2 C\left\langle\delta z \delta p_{\rho}\right\rangle-2 D\left\langle\delta \rho \delta p_{\rho}\right\rangle,
\end{aligned}
$$

with

$$
\begin{aligned}
& A=\frac{\partial^{2} H}{\partial z^{2}}, \quad B=\frac{\partial^{2} H}{\partial z \partial p_{\varphi}}, \quad C=\frac{\partial^{2} H}{\partial z \partial p_{\rho}}, \\
& D=\frac{\partial^{2} H}{\partial \rho^{2}}, \quad E=\frac{\partial^{2} H}{\partial \varphi \partial \rho} .
\end{aligned}
$$

\footnotetext{
${ }^{a)}$ Electronic mail: hbatelaa@unlserve.unl.edu

${ }^{1}$ W. Gerlach and O. Stern, "Der Experimentelle Nachweis der Richtungsquantelung im Magnetfeld," Z. Phys. 9, 349-352 (1922).

${ }^{2}$ N. F. Mott, "The scattering of fast electrons by atomic nuclei," Proc. R. Soc. London, Ser. A 124, 425-442 (1929).

${ }^{3}$ W. Pauli, Magnetism (Gauthier-Villars, Brussels, 1930), pp. 183-186, 217-226, 275-280.

${ }^{4}$ B. M. Garraway, and S. Stenholm, "Observing the spin of a free electron," Phys. Rev. A 60, 63-79 (1999), and references therein.

${ }^{5} \mathrm{~L}$. Brillouin, "Is it possible to test by a direct experiment the hypothesis of the spinning electron?," Proc. Natl. Acad. Sci. U.S.A. 14, 755-763 (1928). ${ }^{6}$ W. Pauli, Collected Scientific Papers, edited by R. Kronig and V. F. Weisskopf (Wiley, New York, 1964), Vol. 2, pp. 544-552.
} 
${ }^{7}$ H. Batelaan, T. J. Gay, and J. J. Schwendiman, "Stern-Gerlach effect for electron beams," Phys. Rev. Lett. 79, 4517-4521 (1997).

${ }^{8}$ G. A. Gallup, H. Batelaan, and T. J. Gay, "Quantum-mechanical analysis of a longitudinal Stern-Gerlach effect," Phys. Rev. Lett. 86, 4508-4511 (2001).

${ }^{9}$ J. H. Shirley, "Validity of the semiclassical approximation in maser theory," Phys. Rev. 181, 600-609 (1969).

${ }^{10}$ M. E. Goggin, B. Sundaram, and P. W. Milonni, "Quantum logistic map," Phys. Rev. A 41, 5705-5708 (1990).

${ }^{11}$ B. Sundaram and P. W. Milonni, "Chaos and low-order corrections to classical mechanics or geometrical-optics," Phys. Rev. E 51, 1971-1982 (1995).

${ }^{12}$ K. Gottfried, Quantum Mechanics (Benjamin, New York, 1966), pp. 6670.

${ }^{13}$ Geoffrey P. Beaumont, Probability and Random Variables (Horwood, Chichester, 1986), p. 222.

${ }^{14}$ Leonard I. Schiff, Quantum Mechanics (McGraw-Hill, New York, 1955), 2nd ed., p. 27.
${ }^{15}$ W. H. Zurek and J. P. Paz, "Decoherence, chaos, and the second law," Phys. Rev. Lett. 72, 2508-2511 (1994).

${ }^{16}$ Siegfried Flügge, Practical Quantum Mechanics (Springer-Verlag, New York, 1971), pp. 101-107.

${ }^{17}$ Claude Cohen-Tannoudji, Bernard Diu, and Franck Laloë, Quantum Mechanics (Wiley, Hermann, Paris, 1977), Vol. 1, pp. 62-64.

${ }^{18} \mathrm{C}$. Leubner, M. Alber, and N. Schupfer, "Critique and correction of the textbook comparison between classical and quantum harmonic oscillator probability densities," Am. J. Phys. 56, 1123-1129 (1988).

${ }^{19}$ Erwin Schrödinger, "Der stetige Ubergang von der Mikro- zur Macromechanik," Naturwissenschaften 14, 664-666 (1926).

${ }^{20} \mathrm{D}$. Styer, "The motion of wave packets through their expectation values and uncertainties," Am. J. Phys. 58, 742-744 (1990).

${ }^{21} \mathrm{H}$. A. Gersch, "Time evolution of minimum uncertainty states of a harmonic oscillator,” Am. J. Phys. 60, 1024-1030 (1992).

${ }^{22}$ Reference 15 , pp. $559-567$.

${ }^{23}$ D. ter Haar, Selected Problems in Quantum Mechanics (Academic, New York, 1964), 2nd ed., pp. 9, 113-114.

${ }^{24}$ Reference 17, pp. $742-764$. 